\title{
Students' media competence formation in French foreign language teaching in the practice of higher education
}

\section{[Formation de la competence mediatique chez les etudiants en classe du francais langue etrangere dans la pratique de l'enseignement superieur]}

\author{
Larisa E. Babushkina - Olga A. Kalugina
}

DOI: $10.18355 /$ XL.2020.13.01.23

\begin{abstract}
The research examines the theoretical issues of the students' media competence formation in French foreign language teaching in the practice of higher education as an interdisciplinary concept that requires a conceptual study and methodological basis. The study revealed the essence, formed the definition of this phenomenon, showed up its structure. The essence and content of the concept of "media competence" are analyzed, the psycholinguistic features of students are revealed, and the possibilities of using media supports are examined. The analysis of the educational opportunities of media supports is carried out and an algorithm for organizing the work of students to create media supports for educational purposes is developed.
\end{abstract}

Key words: media competence, media competence formation, French as a foreign language, higher education practice, media competence formation approaches, media supports

\begin{abstract}
Résumé
La recherche examine les questions théoriques de la formation de la compétence médiatique chez les étudiants en classe du français langue étrangère (FLE) dans la pratique de l'enseignement supérieur en tant que concept interdisciplinaire dont l'étude conceptuelle est une base méthodologique. L'essence et le contenu du concept de «compétence médiatique» sont analysés, les caractéristiques psycholinguistiques des étudiants sont révélées et les possibilités d'utiliser des supports médiatiques sont examinées. L'analyse des possibilités pédagogiques des supports médiatiques est réalisée et un algorithme permettant d'organiser le travail des étudiants en vue de créer des supports médiatiques pour les objectifs pédagogiques est développé.

Mots clés: la compétence médiatique, la formation de compétence médiatique, le français langue étrangère (FLE), la pratique de l'enseignement supérieur, les approches de la formation de compétence médiatique, les supports médiatiques
\end{abstract}

\section{Introduction}

La pertinence du sujet de la recherche.

En considérant l'influence croissante des technologies et des outils multimédias sur l'espace éducatif mondial, ainsi que la nécessité de modifier les formes, les méthodes et les approches de la formation professionnelle des étudiants (Birova et al., 2016), on peut conclure qu'il est important d'étudier les questions liées à la formation de la compétence médiatique chez les étudiants visant à améliorer la qualité de leur formation, éducation et développement aux différents niveaux de l'enseignement. Cet article est consacré à l'étude du problème de la formation de la compétence médiatique des étudiants en classe du français langue étrangère (FLE) dans la pratique de l'enseignement supérieur. Étant donné que la société est informatisée et que les technologies de l'information et de la communication (TIC) sont activement utilisées dans le domaine de l'enseignement, l'attention de la science pédagogique sur les questions relatives à l'éducation aux médias est considérablement intensifiée. 
L'enseignant est chargé d'enseigner aux étudiants à naviguer dans un grand flux d'informations, à synthétiser et à analyser cette information. Ainsi, la formation de la compétence médiatique chez les étudiants en classe du FLE dans la pratique de l'enseignement supérieur devient de plus en plus importante. L'utilisation de produits médiatiques est l'un des moyens les plus efficaces de former la compétence médiatique dans la pratique de l'enseignement supérieur. Le travail indépendant avec ces types d'informations, ainsi que la création directe de supports divers par les étudiants eux-mêmes, seront particulièrement productifs.

Une obligation de l'acquisition de haut niveau de la culture médiatique par des citoyens russes, du développemant de leur capacité à utiliser les produits médiatiques pour leur accomplissement personnel, est cochée dans plusieurs programmes et documents gouvernementaux tels que: la loi fédérale «Sur l'éducation dans la Fédération de Russie»; le programme d'État «Société de l'information 2011 2020»; le concept du développement socio-économique à long terme de la Fédération de Russie pour la période jusqu'à l'an 2020, etc.

L'importance d'une activité responsable, créative et socialement positive des étudiants, la manifestation de leur activité créative et de leur initiative dans l'espace médiatique moderne pour la formation d'une perspective de vie décente sont soulignées dans la Stratégie d'action nationale en faveur des enfants pour 2012-2017. La nouvelle génération des normes éducatives de l'État pour l'enseignement public supérieur comprend déjà l'exigence relative à la formation des compétences chez les étudiants aux médias et au soutien technique des institutions de l'enseignement supérieur. Il est supposé que les étudiants, eux-mêmes, doivent être capables de créer des produits médiatiques réels et utiles pour les organisations éducatives, tout en ayant les connaissances nécessaires des lois de la perception sonore, et de la communication interculturelle ainsi que le concept d'infoéthique.

L'utilisation du potentiel éducatif des ressources médiatiques pour s'épanouir et se réaliser concrétise les problèmes posés par l'éducation d'une attitude responsable et sûre à leur égard, et la réalisation d'un niveau élevé de la culture médiatique personnelle par les adolescents devient l'une des conditions de leur autosuffisance potentielle et de leur évolution de carrière dans une société saturée de médias. Puisque la créativité et l'éducation aux médias commencent à être considérées comme des sphères interdépendantes de la socialisation d'une personne, la question se pose de mettre à jour les mesures d'éducation spécialement destinées à la jeunesse préconisant une créativité des médias.

En travaillant avec des textes médiatiques, les étudiants forment les mêmes qualités personnelles que celles que l'on retrouve dans la pédagogie traditionnelle lors de la lecture des livres (N.B. Kirillova, N.F. Khilko, etc.). Si nous tenons compte du fait que dans la société de l'information, le texte médiatique affecte les valeurs morales et éthiques et les valeurs culturelles de la jeune génération, apprendre à «lire» un tel texte, à percevoir le discours médiatique dans son ensemble et à produire ses équivalents éducatifs est une tâche urgente de l'enseignement supérieur.

L'expérience montre toutefois que jusqu'à présent, l'activité indépendante des étudiants en train de former leur compétence médiatique est l'un des domaines de pédagogie les moins développés. Au début du XXIe siècle, des thèses de doctorat ont été soutenues dans divers domaines liés à l'éducation aux médias: histoire de l'art, études culturelles, sociologie de la culture, vie spirituelle, pédagogie, philologie, philosophie, etc.

En analysant la création de supports pédagogiques par les étudiants en tant que processus constitutif de leur culture médiatique, nous nous appuyons sur une expérience pertinente de l'histoire de la pédagogie nationale et des pratiques modernes. En particulier, sur: les résultats de la recherche liée aux problèmes de l'éducation cinématographique des écoliers, à son lien avec l'éducation spirituelle et

XLinguae, Volume 13 Issue 1, January 2020, ISSN 1337-8384, eISSN 2453-711X 
morale de l'individu; sur les programmes des cours pour les écoliers "Fondements de la culture de l'écran" et "Éducation aux médias", publiés en 1974 et 1985; sur le projet de norme d'éducation aux médias élaboré en 1998-1999; sur le modèle d'éducation aux médias de Baranova, etc.(2002)

Cependant, cette expérience est insuffisante, en particulier pour analyser la phénoménologie de la compétence médiatique et pour l'interpréter comme un concept exprimant la qualité personnelle d'une personne, car les définitions existantes de la compétence médiatique ne prennent pas suffisamment en compte les caractéristiques psychologiques des étudiants. La justification de la structure et du contenu des composantes de la compétence médiatique, des indicateurs de sa formation parmi les étudiants qui étudient le FLE, reste très problématique. La cohérence des indicateurs de perception et d'interprétation identifiés par Fedorov (2005) pour évaluer la compétence médiatique de l'individu, ainsi que l'opportunité d'identifier un indicateur de contact nécessitent une analyse particuliée. Dans la pratique de l'enseignement supérieur la difficulté consiste à envisager des approches pour l'application du processus de création de supports pédagogiques par les étudiants. Ce qui est important, c'est l'analyse approfondie de la relation entre les approches culturelle, d'activité, centrée sur l'élève et environnementale avec l'approche sémiotique, qui n'est pas encore bien étudiée dans les sciences pédagogiques (Birova et al., 2017).

Il ressort de ce qui précède que le problème de notre recherche «La formation de la compétence médiatique des étudiants en classe du français langue étrangère (FLE) dans la pratique de l'enseignement supérieur» est actualisé par les contradictions suivantes: entre la portée scientifique de l'interprétation du terme «compétence médiatique» et la nécessité de clarifier ses composantes pédagogiques afin de l'utiliser dans la pratique de l'enseignement supérieur; entre l'expérience de familiarisation des étudiants avec la créativité des médias et les tendances actuelles en matière d'éducation aux médias et le développement de la société de l'information; entre le potentiel du processus de création par les étudiants de supports pédagogiques médiatique et le manque du développement de méthodes pour la formation de la compétence médiatique des étudiants en classe du français langue étrangère (FLE) dans la pratique de l'enseignement supérieur; entre la pratique pédagogique traditionnelle de l'éducation aux médias des étudiants, qui comprend la visualisation, l'analyse, l'intégration du produit médiatique dans le processus éducatif et la pratique novatrice, impliquant les activités indépendantes des équipes créatives des jeunes pour créer leur propre contenu multimédia éducatif.

La pertinence du sujet, les contradictions identifiées nous ont permis de formuler le problème de la recherche: dans quelles conditions dans le processus de création des supports pédagogiques médiatiques la compétence médiatique des étudiants se forme en classe du français langue étrangère (FLE) dans la pratique de l'enseignement supérieur.

L'objectif de cette recherche est d'examiner les moyens de former la compétence médiatique des étudiants en classe du français langue étrangère (FLE) dans la pratique de l'enseignement supérieur.

L'objet de la recherche est le processus de la formation de lacompétence médiatique des étudiants en classe du français langue étrangère (FLE) dans la pratique de l'enseignement supérieur.

Le sujet de la recherche est le matériel multimédia utilisé comme moyen de former la compétence médiatique des étudiants en classe du français langue étrangère (FLE) dans la pratique de l'enseignement supérieur.

Pour atteindre cet objectif, il est nécessaire de résoudre systématiquement les tâches de recherche suivantes: étudier l'essence et le contenu de la compétence médiatique des étudiants en classe du français langue étrangère (FLE) dans la pratique de l'enseignement supérieur; examiner les caractéristiques psychologiques des étudiants à la première étape de l'éducation dans la pratique de l'enseignement 
supérieur; déterminer le potentiel pédagogique des supports médiatiques dans le processus d'apprentissage en classe du français langue étrangère (FLE) dans la pratique de l'enseignement supérieur; déterminer les spécificités de la formation de la compétence médiatique des étudiants dans le processus de création de supports médiatiques par eux-mêmes en classe du français langue étrangère (FLE) dans la pratique de l'enseignement supérieur; proposer des recommandations sur l'utilisation et la création de supports médiatiques en classe du français langue étrangère (FLE) dans la pratique de l'enseignement supérieur.

L'hypothèse de la recherche: la formation de la compétence médiatique des étudiants dans le processus de création de supports médiatiques par eux-mêmes en classe du français langue étrangère (FLE) dans la pratique de l'enseignement supérieur sera efficace si:

1) dans le travail avec de produits médiatiques, des attitudes de valeur sur la perception des informations des médias se forment en vue du développement personnel des qualités spirituelles, morales et intellectuelles d'un individu;

2) lors de la création d'un produit médiatique, les étudiants sont inclus dans le processus technologique, ce qui est assuré par l'interdépendance des approches culturelle, d'activité, centrée sur l'élève et environnementale dans la conception du contenu de ce processus;

3) les activités des étudiants dans la création d'un produit médiatique leur permettent de modéliser et de refléter leur propre vision du matériel éducatif, tout en adaptant leur potentiel créatif personnel au monde des médias;

4) l'interaction pédagogique en forme de dialogue, la prise en compte des intérêts, les manifestations individuelles et les capacités créatrices des étudiants sont assumées dans le processus de création de supports pédagogiques médiatiques.

\section{Revue de litérature}

La recherche a été réalisée sur la base théorique de l'étude des travaux suivants:

- principales questions de la théorie moderne et de la méthodologie d'enseignement d'une langue étrangère (Arutyunova, 2019; Bim, 1988; Kashchuk, 2019; Kostina \& Pronkina,2015, Shukshina \& Babushkina, 2014; Smirnova \&Bagramova, 2018; Solntseva2019 ; Vetoshkin, 2015; Yakunchev \& Gorshenina, 2013; Zimnyaya, 2003; Masalimova, et al. 2019.).

- concepts d'enseignement,centrée sur l'apprenant (Barievaet al., 2018; Hutorskoj, 2002; Kozarenko, 2019; Shukshina et al. 2016; Ulil \& Sahril, 2018);

- idées sur l'informatisation de l'éducation (Lazutova, Falileev, 2016 ; Levina \& Kostina, 2016, Borisova, et al., 2016).

- concepts des technologies de l'information et de la communication (TIC), supports pédagogiques médiatiques pour le développement de l'intelligence des étudiants (Kadakin et al. 2017);

- théorie et pratique de l'organisation du processus éducatif de l'enseignement supérieur sur la base de supports pédagogiques médiatiques (Kadakin et al., 2016 ; Saienko et al., 2019);

- théorie de l'apprentissage dans la société de l'information (Fedorov, 2005);

- points de vue sur la formation d'une culture de l'information personnelle (Gnezdilova \& Zaitsev, 2018; Gurevich, 2011, etc.);

- théorie esthétique de l'éducation aux médias (Chevallier, 1980; Federman, 1997);

- théorie pratique de l'éducation aux médias (Balakishiyeva, 2017, Baranov, 2002 ; Muzykantet al., 2019);

• théories de "la pensée critique" (Freinet, 1994).

XLinguae, Volume 13 Issue 1, January 2020, ISSN 1337-8384, eISSN 2453-711X 


\section{Matériaux et Méthodes}

Pour résoudre les problèmes de cette recherche les méthodes suivantes ont été utilisées:

- analyse théorique de la littérature psychologique, pédagogique et scientifiquement méthodique, du matériel de recherche scientifique;

- étude et synthèse d'expérience pédagogique;

- activités d'évaluation et de contrôle de l'efficacité du travail avec du matériel médiatique en formant la compétence médiatique des étudiants en classe du français langue étrangère (FLE) dans la pratique de l'enseignement supérieur.

Aspects théoriques de la formation de la compétence médiatique des étudiants dans le processus de création de supports médiatiques par eux-mêmes en classe du français langue étrangère (FLE) dans la pratique de l'enseignement supérieur

À l'heure actuelle, la société impose à une personne des exigences particulières : éducation, dévouement, sens des relations interpersonnelles, capacité de travailler en équipe et, plus important encore, capacité de naviguer dans un énorme flux d'informations, de l'analyser et de le synthétiser, ainsi que de le mettre en pratique. L'enseignement supérieur a pour tâche de préparer une telle personne, de sorte que les normes régissant l'enseignement subissent actuellement des changements importants et changent de priorités.

L'expérience d'enseignement supérieur moderne montre que les étudiants maîtrisent mal le travail avec l'information et que les technologies et les méthodes visant à la formation et au développement de compétence médiatique en classe acquièrent une pertinence particulière. Ainsi, il devient nécessaire de résoudre le problème de la formation de la compétence médiatique des étudiants en classe du français langue étrangère (FLE) dans la pratique de l'enseignement supérieur.

Tout d'abord, ça vaut la peine d'être déterminée ce que c'est la compétence médiatique en général. Le concept de «compétence médiatique» se compose de deux parties : «médias» et «compétence».

Média (du latin "media", "medium" signifie"moyen","médiateur") est un terme introduit à l'origine pour faire référence au phénomène de "culture de masse" ("mass culture", "mass media"). C'est un type de culture de la société de l'information qui fait partie du concept de culture générale et qui est représenté par des publications imprimées telles que : livres, journaux, magazines, cinéma, radio et télévision, ressources d'Internet, tout ce qui relie une personne au monde extérieur, informe, divertit, promeut, a un impact sur les évaluations, les opinions et le comportement des personnes (Bim, 1988).

Une attention particulière devrait être portée à l'essence du terme «compétence» et à ses caractéristiques concernant l'apprentissage d'une langue étrangère. P.S. Gurevich interprète le concept de "compétence" comme "... la capacité à intégrer les connaissances et les maîtrises à leur utilisation dans l'environnement externe" (Gurevich, 2011: 148). Selon Zimnyaya (2003), Hutorskoj (2002) le terme «compétence» comprend un ensemble de compétences pertinentes d'une personne et son attitude personnelle à l'égard du sujet de son activité. Selon ces chercheurs, le concept de "compétence" devrait être compris comme un ensemble de qualités personnelles interdépendantes, prédéterminées par rapport à un certain ensemble d'objets, de processus et de phénomènes qui sont nécessaires pour réaliser des activités productives de haute qualité.

Les compétences sont les principales unités d'évaluation de la qualité et des résultats de l'apprentissage d'une langue étrangère. Dans la littérature de recherche, ce concept est abordé sous différents angles, ce qui est associé à la complexité de la structure de l'activité professionnelle dans divers domaines. La compétence agit comme un concept intégré, alliant les qualités personnelles de l'individu grâce à l'expérience professionnelle des activités d'une personne. La compétence est un 
ensemble des qualités personnelles de l'individu en action. Nous estimons que la compétence médiatique des étudiants est un ensemble de leurs qualités professionnelles, médiatiques et créatives.

Il convient des ouligner une grande diversité de terminologie dans la recherche scientifique liée au concept de «compétence médiatique». De nombreux chercheurs (Baranov, 2002, Fedorov, 2005, Hutorskoj, 2002, etc.) ont analysé différentes définitions et ont découvert que la terminologie non normalisée était utilisée dans le monde moderne. Par conséquent, tant dans les études étrangères que celles nationales sur ce sujet, des termes courants tels que «éducation aux médias», «information», «informatique», «multimédia», «éducation aux médias» et «éducation audiovisuelle» sont souvent utilisés comme synonymes (Fedorov, 2005: 82).

L'origine des idées sur la compétence médiatique en Europe est liée à l'émergence de la presse et du cinéma dans les années 20-30 du XX siècle. Les études de S. Freinet (1994) sont particulièrement intéressantes. C'est un pédagogue français qui associe avec succès le pédocentrisme de la pédagogie européenne et le travail créatif dans la rédaction et l'imprimerie lors de la création de journaux scolaires en dehors des horaires des classes. S. Freinet (1994) a examiné le développement de la compétence médiatique dans le processus de création de «textes libres» en tant qu'article de journal. Par conséquent, depuis le début de l'éducation aux médias, l'essence même de la compétence médiatique avait un lien direct avec le processus de réalisation de la personnalité face aux médias, ce qui induit une transformation du rôle historiquement établi du créateur et amène le processus de développement de la compétence médiatique à l'invention d'un nouveau plan de pensée et à la mobilisation de l'information, caractéristiques de la position de pragmatisme dans la philosophie étrangère (Freinet, 1994).

L'avis de S. Freinet, qui ne considérait le cinéma et la photographie que comme un divertissement et un moyen d'apprentissage, était particulièrement remarquable. Il était «une manifestation d'une nouvelle forme de pensée et d'expression» (Freinet, 1994: 82). S. Freinet (1994) est convaincu que les participants au processus éducatif «devraient maîtriser le langage des médias audiovisuels» par analogie avec la connaissance pratique de base de la peinture, car «si vous ne dessinez pas vous-même, mais regardez le tableau, c'est une seule chose, mais si vous pouvez dessiner vous-mêmes, donc vous pouvez apprécier mieux le travail d'un autre artiste» (Freinet, 1994: 51). Les idées de S. Freinet (1994) ont formé les conditions préalables à l'introduction des débuts créatifs personnels d'un étudiant et d'un enseignant dans le processus de création de ressources médiatiques. Il convient également de noter qu'en tant qu'artiste, tout en travaillant sur une image, il crée son propre espace de création personnel, de même que le créateur d'une ressource médiatique doit pouvoir créer son propre espace d'informations reflétant des aspects personnels. Cette idée de créer une ressource médiatique place ce processus au niveau de la créativité, ce qui forme les conditions préalables à l'émergence d'idées sur la compétence médiatique des étudiants en classe d'une langue étrangère dans la pratique de l'enseignement supérieur. Il est évident que la création de l'environnement pédagogique médiatique par un enseignant est une condition préalable à la création réussie d'une ressource médiatique créée par un étudiant.

Vers les années 1970, l'introduction de l'éducation aux médias dans les écoles supérieures ont été activement mises en œuvre, ce qui a permis de prendre en compte la personnalité de l'enseignant à compétence médiatique. Par exemple, en France, en 1975, l'Institut de formation pour le développement de la culture cinématographique a été créé. Il prévoyait l'impact de l'éducation aux médias sur les universités. La tâche de former des éducateurs qualifiés aux médias a atteint un niveau de priorité, ce qui a permis de rapprocher le domaine d'influence de l'éducation aux médias vers le système de perfectionnement professionnel. Au niveau du ministère

XLinguae, Volume 13 Issue 1, January 2020, ISSN 1337-8384, eISSN 2453-711X 
dans le système éducatif français, des médias étaient considérés comme une «école parallèle» (terme introduit par Federman (1997) en tant que traducteur d'éléments de connaissance, souvent différents de ceux d'un enseignant ou d'un manuel. Au niveau fédéral, il a été noté que l'apprentissage par les médias devrait être pris en compte par l'école classique.

J. Chevallier (1980) note que le nombre de tâches d'éducation aux médias créées en 1977 pour les éducateurs aux médias, ainsi que la compréhension et l'évaluation esthétique du contenu des ressources médiatiques comprenaient: «amener les étudiants à créer leurs propres courts métrages basés sur l'art de la photographie; choisir des passages musicaux avec des étudiants pouvant accompagner certains épisodes ; dispenser une formation pratique sur les enregistrements sonores» (Chevallier, 1980: 27). En 1978, ces tâches ont été complétées par «l'inclusion des étudiants dans le tournage de leurs propres reportages vidéo et photographiques; au niveau pratique, organiser la création de petits documents vidéo par les étudiants euxmêmes» (Chevallier, 1980: 34). En conséquence, le début des années 70 a été marqué par le début de la fusion des orientations esthétiques, sémiotiques et activités de l'éducation aux médias dont les conditions préalables à l'émergence d'idées sur la compétence médiatique de l'enseignant ont été activement mises en œuvre dans le processus de création artistique indépendante avec les médias. On peut dire qu'en raison de l'influence des composantes de la connaissance et de l'esthétique sur le processus de création des ressources médiatiques, la personnalité se développe dans les résultats des activités, qui peuvent à ce stade être considérées comme une compétence médiatique de l'enseignant.

Sur la base de ce qui précède, on peut distinguer les caractéristiques de la compétence médiatique, ainsi que ses formes : l'utilisation de produits médiatiques dans n'importe quel domaine de la vie, l'absence de frontières territoriales, la simultanéité de la synthèse auditive, visuelle, verbale, même olfactive et gustative dans les médias modernes, la formation d'un type de pensée fondamentalement différent aussi que des principes de la perception interactive de l'information dans la culture médiatique, l'altérité des relations entre l'auteur et le lecteur, aussi qu'entre le producteur et le consommateur.

La compétence médiatique est un phénomène multifonctionnel. Un certain nombre de chercheurs distinguent les fonctions suivantes de la compétence médiatique:

- la fonction informative, grâce à laquelle l'information sociale est stockée et accumulée dans la société à l'aide des outils créés par l'humanité;

- la fonction communicative, grâce à laquelle l'information est échangée;

- la fonction normative ou idéologique, grâce à laquelle la compétence médiatique est responsable du processus de socialisation de l'individu, de l'assimilation de l'expérience sociale, des normes, des connaissances, des idéaux, correspondant à une telle ou telle société;

- la fonction détente ou divertissantequi est associée aux besoins de l'homme en repos mental et physique; grâce àcette fonction la compétence médiatiqueoffre de nombreuses possibilités de relaxation: télévision, internet, jeux informatiques, etc.

- la fonctioncreative qui permet de satisfaire la curiosité dans n'importe quel domaine de la vie, de prendre part au développement et à la transformation du monde;

- la fonctionintégrativegrâce à laquellela compétence médiatiquefavorise l'unification d'unpeuple, conserve tout ce qui est accumulé par l'État, crée un lien entre les générations;

- la fonctionintermédiairegrâce à laquellela compétence médiatiqueétablit un lien entre les structures de la société.

La compétence médiatiquedes étudiants s'exprime sous forme d'indicateurs de contact, de motivation, de conceptualité, d'évaluation et de création. Le problème de la formation de compétence médiatiquedes étudiants est particulièrement actuelle, 
en raison du développement rapide des technologies de l'information et de la communication (TIC). Il permet aux étudiants de naviguer dans le monde des médias, de percevoir, d'analyser, d'évaluer et de mettre en pratique de manière adéquate les données obtenues des médias, d'Internet, des films, etc.

La compétence médiatique des étudiants est une combinaison de niveaux de développement personnel, c'est à dire la capacité depercevoir le texte du media, d'analyser le texte du media, d'évaluer le texte du media, de faire preuve de créativité dans les medias, d'acquérir de nouvelles connaissances dans le domaine des médias.

Ainsi, la formation de la compétence médiatique de la jeune génération devient l'une des priorités dans le domaine de l'éducation. Aujourd'hui, il est nécessaire non seulement de donner des connaissances toutes faites, mais aussi faire savoir comment cultiver une culture de leur formation, de leur changement et de leur transformation. Ces problèmes peuvent être résolus grâce à l'éducation aux médias, c'est-à-dire en préparant les étudiants à l'utilisation des médias.

\section{Caractéristiques psycholinguistiques des étudiants en début d'apprentissage de l'enseignement supérieur}

La société revendique la formation d'une personnalité d'une éducation moderne qui sait résoudre les différentes tâches à la manière indépendante, penser de manière critique, développer et défendre son point de vue et ses croyances, renouveler et mettre à jour en permanence ses connaissances grâce à l'auto-éducation, améliorer ses compétences et les appliquer de manière créative.

L'une des tâches principales d'un enseignant de langues étrangères est d'enseigner à apprendre. Pour cela, il est nécessaire de créer des conditions permettant aux étudiants de chercher consciemment à maîtriser de nouvelles connaissances.

L'enseignement d'une langue étrangère aux étudiants se fait à la fois sous la direction d'un enseignant et au cours d'un travail indépendant.

Pour que la formation se déroule avec succès, il est nécessaire de prendre en compte un certain nombre de caractéristiques psychologiques et linguistiques des étudiants.

L'attention est l'un des principaux domaines d'activité mentale des étudiants. Il nous permet de comparer, de peser et d'examiner soigneusement les parties du tout, d'associer une nouvelle impression à l'ancienne.

C'est à ce titre-là que la question se pose: comment stimuler l'activation de l'attention pendant l'enseignement?

Tout d'abord, les étudiants devraient être intéressés par la matière qu'ils étudient. L'enseignant doit veiller à ce que le matériel, d'une part, ne perde pas en nouveauté, de l'autre part, soit accessible à la perception.

En effet,quandl'attention de l'étudiant devient arbitraire et il peut se concentrer sur la résolution de problèmes plus longtemps, le matériel sera absorbé encore plus rapidement.

La mémoire est le processus psychologique et physiologique le plus important qui détermine le développement et le progrès intellectuel de l'individu.

Selon les recherches scientifiques, la base des mécanismes de la mémoire à court et à long terme est la même, mais il existe encore quelques divergences, par exemple, avec la manifestation de la mémoire à long terme certains changements morphologiques se produisent dans l'appareil des neurones.

La manifestation de ces changements est stimulée par de multiples exercices. Par conséquent, une révision et des exercices de consolidation des informations reçuessont des conditions préalables à la transition du niveau de la mémoire à court terme au niveau de la mémoire à long terme. C'est pourquoi la tâche principale d'un enseignant de langues étrangères est l'organisation rationnelle de la révision du matériel.

XLinguae, Volume 13 Issue 1, January 2020, ISSN 1337-8384, eISSN 2453-711X 
Lorsqu'on organise une révision, on doit connaître avec quelle vitesse on oublie l'information reçue.

Selon les psychologues, le processus d'oubli est très intense, en particulier au début de l'assimilation d'une langue. Par exemple, dans la littératureil y a des données suivantes: environ $40 \%$ du matériel est oubliépendant une demi-heure après la présentation; environ 34\%du matériel est oubliéle lendemain; environ $21 \%$ du matériel est oubliéaprès 30 jours (Bim, 1988).

Faisons également attention au fait qu'il existe trois types de mémoire impliqués dans la classe du français langue étrangère: mémoire acoustique, mémoire visuelle et mémoire sensiomotrice. L'enseignant doit faire attention au type de mémoire qui prévaut chez l'étudiant.

La forme graphique du mot est crucialepour les étudiants qui ont une bonne mémoire visuelle. Ils représentent visuellement ce qui est écrit à la main et en parlant ils lisent ces motsdans leur esprit. Mais la connaissance pratique de la langue est davantage associée à la mémoire acoustique. Par conséquent, un étudiant qui n'a développé qu'une mémoire visuelle apprend lentement la langue française. Il est difficile pour lui de mémoriser le son et l'intonation. Sa prononciation est respectivement mauvaise. Et au contraire, il apprend facilement la grammaire, car il visualise immédiatement les terminaisons.

Les étudiants pensent en utilisant des images sonores ayant la mémoire acoustique développée. Les deux autres types de mémoire jouent un rôle complémentaire. Un étudiant qui possède une bonne mémoire acoustiqueapprend une langue étrangère plus facilement qu'un étudiant avec une mémoire visuelle développée. Il se souvient facilement des mots entrés oralement, mais pas de différentes formes grammaticales, telles que la terminaison ou la déclinaison.

Un étudiant avec une mémoire sensiomotricebien développée penseplus facilement pardes images apprises à la suite d'une activité motrice. Les images sonores et visuelles n'ont aucune signification pour lui. En lisant, il perçoit une à une les syllabes et les mots, ressentant clairement les mouvements effectués lors de l'articulation active de ces mots. Possédant ce type de mémoire il est le plus difficile pour un étudiant à apprendre une langue étrangère. Mais, Selon les chercheurs ce type de mémoire est rare.

Par conséquent, on peut conclure que la formation utilisant du matériel vidéo sera plus efficace car presque tous les types de mémoire sont impliqués. Les étudiants peuvent à la fois percevoir des informations à l'aide de supports vidéo et créer leurs propres supports vidéo.

Depuis l'adolescence, l'étudiant essaie d'exprimer son désir d'affirmation, d'expression de lui-même, de capacité de défendre ses points de vue et ses convictions avec une force particulière. Les facteurs qui, à ce niveau d'apprentissage d'une langue étrangère, revêtent une importance particulière, sont l'orientation communicative croissante de l'apprentissage d'une langue étrangère et la création d'un climat psychologique favorable à la communication, ainsi que l'orientation professionnelle. D'où le choix du matériel, sa nature problématique, stimulant les échanges d'opinions, encourageant la réflexion, le choix viennent. À cet âge, le désir d'indépendance se manifeste avec une urgence particulière, et l'enseignant doit examiner attentivement son style de communication avec les étudiants (Fedorov, 2005).

Dans les conditions où l'étudiant enseigne une langue étrangère, il est nécessaire de s'assurer qu'il est intéressé par la réussite d'un cours de langue étrangère. De toute évidence il faut s'appuyer sur la conscience, l'orientation créative, l'indépendance, l'activité, les intérêts cognitifs, y compris les intérêts professionnels, pour former sa personnalité de toutes les manières possibles, pour la développer de manière globale au moyen d'une langue étrangère (Balakishiyeva, 2017).

La prise en compte de toutes les caractéristiques psychologiques dans l'enseignement d'une langue étrangère contribue sans aucun doute à créer un effet 
positif dans la formation de la culture médiatique et l'organisation du travail avec du matériel vidéo.

\section{Résultats et discussion}

Les conditions pour la formation de la compétence médiatique des étudiants dans le processus de création de supports médiatiques par eux-mêmes en classe du FLE dans la pratique de l'enseignement supérieur

Dans les conditions de la médiatisation rapide de la société moderne, du développement de TIC, de la redondance et de la confusion des flux d'informations, de la disponibilité générale et de la prévalence de divers types de technologies, les adolescents ont non seulement pu utiliser n'importe quelle ressource d'Internet, mais aussi créer et placer facilement leurs sites Web, blogs, microblogs, photos sur Internet, du matériel audio et vidéo, pour former divers types de communautés sur Internet, pour mener des activités en réseau. Ces circonstances indiquent le besoin urgent de formation de la compétence médiatique des étudiants, étroitement liées à un champ d'information.

Les adolescents modernes ne peuvent plus se passer de films, de télévision, d'Internet, de divers types de réseaux sociaux, de jeux informatiques, etc. Tout cela conduit à un changement d'orientation dans le domaine de l'éducation aussi.

À l'heure actuelle, les étudiants ne doivent pas seulement être capables de naviguer dans un large flux d'informations médiatiques, de sélectionner consciemment les informations pertinentes, d'avoir des compétences pratiques pour travailler avec les médias, mais aussi d'avoir des connaissances audiovisuelles, une pensée créative, une bonne imagination, une perception morale et éthique et un goût artistique.

Sur cette base, l'enseignant est confronté à une tâche importante: apprendre à un adolescent à naviguer dans le flux d'informations médiatiques, lui apprendre à analyser et à synthétiser des informations obtenues à partir de sources médiatiques, et à lui apprendre à créer ses propres supports médiatiques.

En créant un espace pour la réalisation d'idées créatives dans le développement et l'application de contenus audiovisuels modernes dans divers projets (recherche, création, jeux de rôle, orientation, informations) et d'autres activités, les anciens formats sont remplacés par de nouveaux formats de textes vidéo, audio et autres supports, graphismes 3D, sites open source, Web sémantique, éditeurs multiplateformes, téléviseurs et plus.

Cela permet, dans le processus d'interaction pédagogique, de réaliser plus largement les outils et technologies de convergence des supports médiatiques, en communiquant avec les adolescents en tant que partenaires actifs du processus éducatif créatif, en vue de la réalisation des objectifs de l'éducation, de la formation de la compétence médiatique des étudiants.

La création et l'utilisation de divers supports médiatiques dans le processus d'apprentissage permettent à l'enseignant de développer activement de differents types de pensée des étudiants, de créer une conscience esthétique et une expérience de l'activité artistique et créative, d'analyser les causes psychologiques et pédagogiques de l'impact du matériel médiatique sur le développement personnel, de définir leur attitude à l'égard des textes médiatiques perçus.

Afin de posséder des techniques de formation de la compétence médiatique des étudiants, l'enseignant doit maîtriser les technologies suivantes : organisation d'un climat psychologique favorable et d'un système de motivation interne pour travailler avec des fichiers médiatiques ; diagnostics du développement général des étudiants, du déroulement du processus éducatif et de ses résultats ; gestion de l'apprentissage et de l'activité cognitive des étudiants, y compris l'aspect de l'orientation pratique vers la

XLinguae, Volume 13 Issue 1, January 2020, ISSN 1337-8384, eISSN 2453-711X 
créativité indépendante dans le domaine des arts audiovisuels;auto-analyse des résultats d'apprentissage (réflexion).

Également pour une formation réussie de la compétence médiatique des étudiants, il convient de se référer aux normes éducatives de l'État, selon lesquelles l'équipement technique et médiatique du processus éducatif devrait fournir:l'organisation des événements de masse, des réunions, des séminaires, des conférences, des olympiades, des activités récréatives des étudiants qui donnent la possibilité de visionnage de films et de vidéos, ainsi que l'organisation de travaux scéniques, de représentations théâtrales, accompagnés de son, lumière et multimédia;la publication des recherches d'étudiants, des produits médiatiques des étudiants;l'enregistrement, la manipulation d'images et la sonorisation; audio-, videoaccompagnement de projets éducatifs et autres documents axés sur le développement de la culture de l'information et des médias des étudiants en cours facultatifs et complémentaires, élargissant l'utilisation des technologies de l'information et de la communication pour la mise en œuvre de nouvelles formes et méthodes d'enseignement.

L'une des principales caractéristiques de l'activité médiatique de l'étudiant devient celle de créer un texte. Le reste de l'activité médiatique est réalisé avec la conversion d'informations textuelles en un autre type d'information médiatique.

Sur la base de ce qui précède, on peut noter que l'alphabétisation d'un étudiant joue un rôle important dans la création de produit médiatique: il a besoin de lire, d'avoir un discours compétent, d'être ouvert d'esprit, d'être une personne polyvalente et de s'efforcer d'un développement d'épanouissement personnel.

Ainsi, la formation de la compétence médiatique des étudiants devient l'une des priorités dans le domaine de l'éducation. Aujourd'hui, il est nécessaire non seulement de donner des connaissances toutes faites, mais aussi de cultiver une culture de leur formation et de leur changement, de leur transformation et de leur conversion.

Compte tenu des caractéristiques d'âge des étudiants, l'apprentissage du français langue étrangère sera beaucoup plus facile avec du matériel vidéo, car ils contribuent à l'actualisation des connaissances, affectent tous les types de mémoire et sont capables de maintenir l'intérêt des étudiants au bon niveau.

Il convient également de noter que l'établissement d'enseignement et l'enseignant doivent créer certaines conditions pour que les étudiants puissent apprendre le français langue étrangère, ce qui est inextricablement lié à l'utilisation des technologies de l'information et de la communication et implique un changement d'idées sur les activités créatives constructives des étudiants dans le domaine des médias.

Pour former efficacement la compétence médiatique des étudiants en classe du FLE, nous suggérons d'utiliser un ensemble de conditions pédagogiques. L'analyse de la littérature psychologique et pédagogique, l'expérience de l'enseignement supérieur, nous a permis d'identifier les conditions pédagogiques suivantes:

1. Adaptation du niveau individuel de formation de la compétence médiatique et des caractéristiques individuelles des étudiants. Cette condition est exprimée par la mise en œuvre d'une approche individuelle de l'enseignement du FLE, par la création des conditions psychologiques confortables dans un groupe en classe du FLE, par la réalisation d'une surveillance continue de la formation de compétence médiatique des étudiants. La création de cette condition implique le développement d'un système de valeurs des étudiants, de leurs qualités personnelles, d'un accroissement du niveau de leur motivation et de leur activité dans le processus d'apprentissage du FLE.

2. Modélisation du travail indépendant dans différents types d'activités éducatives (analyse, recherche, conception, créativité). Cette condition se traduit par la construction d'un parcours d'apprentissage individuel pour chaque étudiant, par 
l'accomplissement des fonctions de rôle à chaque étape du travail dans l'environnement de l'éducation aux médias, par la liberté de choisir la forme de travail dans l'environnement de l'éducation aux médias et les moyens de créer des produits médiatiques, par l'utilisation de méthodes d'enseignement actives et interactive en classe du FLE. La mise en œuvre de la condition implique une formation intensive de compétences dans l'utilisation des TICs, des compétences professionnelles, médiatiques et créatives des étudiants en classe du FLE.

3. Fonctionnement indépendant dans l'environnement de l'éducation aux médias créé par un enseignant du FLE. Cette condition est liée avec l'immersion de chaque étudiant dans le processus d'activité constructive créative, l'assurance d'une interaction mutuelle des participants dans le processus éducatif dans l'environnement d'éducation aux médias du FLE, en utilisant les TICs, les multimédias, les hypermédias dans la création d'un produit médiatique.

4. Assurance de la coopération de l'enseignant et des étudiants dans le processus de travail avec de diverses resources médiatiques. Cette condition est visée à la formation effective de compétence médiatique des étudiants dans l'enseignement du FLE sous réserve d'une interaction individuelle continue, d'une coopération entre les étudiants et l'enseignant qui joue le rôle de tuteur.

La réalisation de la première condition suppose la création d'un environnement psychologiquement confortable pour des étudiants dans le processus d'apprentissage du FLE, la surveillance continue de leur état émotionnel et le processus de formation de leur compétence médiatique. La formation de compétence médiatique des étudiants en classe du FLE est indissociable de la conception et du fonctionnement de produits médiatiques dans un environnement de l'éducation aux médias créé à partir de divers textes médiatiques au moyen des TICs, du multimédia, etc. Par conséquent, l'activité d'un enseignant de FLE est inextricablement liée à l'immersion des étudiants dans une activité de création constructive des produits médiatiques utilisant les TIC, ce qui se reflète dans la création de la deuxième condition pédagogique. La réalisation des troisième et quatrième conditions implique la formation de compétences professionnelles, créatives et médiatiques des étudiants en cours d'apprentissage du FLE et contribue à l'immersion dans des activités constructives créatives, où les étudiants démontrent leur créativité, réalisent leur expérience professionnelle et personnelle, augmentent le niveau de compétences professionnelles, médiatiques et créatives.

Organisation du travail des étudiants sur la création de supports médiatiques par eux-mêmes en classe du FLE dans la pratique de l'enseignement supérieur

Analyse des capacités éducatives des supports médiatiques. Comme mentionné précédemment, l'utilisation des vidéos vous permet d'organiser le processus éducatif de manière beaucoup plus efficace car ils contribuent à l'actualisation des connaissances et au maintien de la motivation pour apprendre au niveau approprié.

Le terme "vidéo" (du latin "video" signifie "je vois") comprend un large éventail de technologies pour l'enregistrement, le traitement, la transmission, le stockage et la lecture de matériel visuel et audiovisuel sur des moniteurs.

En outre, les supports vidéo sont considérés comme l'un des types de moyens techniques d'enseignement, qui permettent de transmettre des informations et de recevoir des informations en retour dans le processus de perception et d'assimilation de ces informations en vue du développement ultérieur de certaines compétences chez les étudiants en classe du FLE (y compris l'expression orale). Le matériel vidéo utilisé en classe du FLE doit être authentique, c'est-à-dire créés par des locuteurs natifs, alors qu'ils ne sont souvent pas de nature éducative et ne sont pas liés au processus d'apprentissage.

L'expérience pratique montre que l'utilisation de la vidéo est un moyen efficace de développer la pensée verbale des étudiants et de résoudre plusieurs

XLinguae, Volume 13 Issue 1, January 2020, ISSN 1337-8384, eISSN 2453-711X 
problèmes simultanément, ce qui correspond au principe d'une approche intégrée de l'apprentissage. Les étudiants reçoivent une représentation visuelle de la vie, des traditions et des réalités linguistiques des pays francophones. L'utilisation d'enregistrements vidéo contribue à l'individualisation de l'apprentissage et au développement de l'activité de la parole motivée des étudiants. En raison du changement d'impression et de l'impact émotionnel de la vidéo, l'attitude personnelle des étudiants est formée à l'égard de ce qu'ils voient.

Sur la base de la documentation disponible sur Internet, ainsi que de notre propre analyse des services et programmes vidéo existants, nous présentons une brève analyse des plus populaires d'entre eux:

1. Youtube est un site d'hébergement de vidéos qui offre aux utilisateurs la possibilité de stocker, diffuser et afficher des vidéos.

L'avantage incontestable de ce site est qu'il est possible de travailler avec des textes authentiques, d'écouter les paroles de locuteurs natifs et d'entretenir un dialogue avec eux.

Également sur le portail Youtube, on peut trouver une énorme quantité de documents vidéo variés qui peuvent être à la fois divertissants et éducatifs.

Un autre avantage incontestable est l'interactivité. On peut interagir directement avec le créateur de la vidéo, poser des questions, mener une conversation active. On peut également lancer «diffusion en direct», ce qui nous permet de partager les dernières informations.

L'un des inconvénients les plus importants de ce site est l'absence d'options d'édition des vidéos.

2. HyperCam nous permet d'enregistrer des actions à l'écran avec la possibilité de les éditer. Le programme permet de créer facilement et rapidement des présentations vidéo, des vidéos de formation, des clips de démonstration. Il peut être installé à la fois sur un ordinateur personnel et sur un appareil mobile.

Un inconvénient important est que la version complète de ce programme est payée.

3. Rambler Visionest un portail multimédia interactif. Il permet aux utilisateurs du Web d'afficher des travaux auparavant inaccessibles à un large éventail de téléspectateurs. En outre, les auteurs ont la possibilité de présenter leurs œuvres à un large public. À l'heure actuelle dans Rambler Vision il y a cinq sections:

Animation (des dessins de festival uniques en provenance de grands studios d'animation qui sont presque impossibles à voir sur les principales chaînes de télévision).

Art vidéo (une culture vidéo alternative, conceptuelle et expérimentale, cinéma non formaté, annonces et rétrospectives de programmes de festivals).

Clips vidéo (des vidéos musicales, y compris celles non formatées).

Films documentaires (courts métrages de documentaires professionnels.

Cinéma (annonces de grands films).

4. Movie Makerest l'un des éditeurs vidéo les plus populaires qui nous permet de couper et de coller la vidéo, d'ajouter divers effets et la musique. Il est convient à la création de vidéos et à la création de présentations.

\section{Algorithme de la création de supports médiatiques par des étudiants en classe du FLE dans la pratique de l'enseignement supérieur}

Les particularités des activités médiatiques des participants au processus éducatif résident dans le fait que la plupart des projets médiatiques reposent, dans leur mise en œuvre, sur des algorithmes de développement de structure très similaire.

L'algorithme de création de produits médiatiques qui favorise la formation de compétence médiatique des étudiants comprend les composants suivants: analyse, discussion, évaluation de produits tout faits de haute qualité, familiarisation avec la terminologie de l'éducation aux médias ; exercices sur la conversion de supports 
médiatiques d'un type à un autre, détaillant un projet de création de ces produits; recherche d'idées, élaboration d'un scénario de texte médiatique planifié, planification d'un produit médiatique, création de storyboards, modélisation, y compris utilisation de structures graphiques (croquis, graphiques, diagrammes, cartes mentales, etc.), étude de questions théoriques sur le sujet, analyse d'options pratiques des activités disponibles et des ressources nécessaires; préparation des supports médiatiques; analyse du texte d'écran en détails ; création de votre propre prouduit médiatique en tenant compte de vos connaissances théoriques, besoins, intérêts et compétences.

Sur la base de l'algorithme ci-dessus, on invite les étudiants à développer, par exemple, un projet sur le sujet «Problèmes environnementaux».

Objectif du projet: Education de l'environnement et formation d'une culture écologique du comportement des étudiants basée sur le développement professionnel, spirituel et moral de l'individu à travers des activités conjointes des étudiants et des enseignants.

Tâches du projet:former une citoyenneté active de la jeune generation; fromouvoir l'éducation morale, environnementale, esthétique et professionnelle des jeunes; étudier la situation environnementale dans le monde, dans notre pays et dans notre ville; coordonner des activités pratiques des jeunes dans l'étude et l'évaluation de l'état de l'environnement sur l'exemple de notre ville et de ses banlieues;encourager un sens de la responsabilité personnelle des jeunes pour l'environnement;développer l'initiative et la créativité des étudiants à travers l'organisation d'activités socialement importantes; diffuser les connaissances environnementales non seulement dans les internats, mais également parmi la population locale, en utilisant diverses activités et formes de travail.

\section{Étape 1}

Les étudiants sont invités à regarder la vidéo educative aussi que la vidéo sur la création de ce genre de videos, par exemple, sur la chaîne de Youtube: "Comment faire une vidéo";"Animaker Tutorial";"Comment faire une vidéo en ligne".

\section{Étape 2}

Les étudiants sont encouragés à créer leur propre vidéo animée en utilisant le texte proposé sur la base du site Web Animaker et du programme Movie Maker.

\section{Étape 3}

Les étudiants sont encouragés à développer leur propre vidéo sur le sujet «Problèmes environnementaux».

Au début il faut choisir un format vidéo. On propose le format de la vidéo documentaire.

On doit également choisir un programme de traitement des clips. Movie Maker est la meilleure option, car ce programme est facile à utiliser et à télécharger gratuitement sur PC.

Les étudiants préparent un scénario, réfléchissent au lieu et à l'heure du tournage, au scénarimage et à la coupure du texte.

Pour une étude plus approfondie du problème, les étudiants sont invités à analyser du matériel médiatique différants sur ce sujet. 


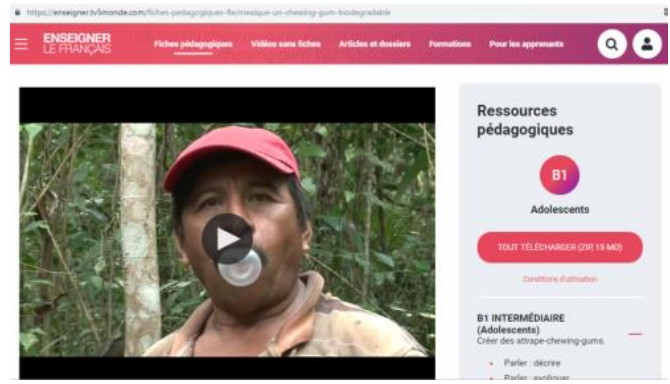

Image 1.Mexique : un chewing-gum biodegradable.

Url: https://enseigner.tv5monde.com/fiches-pedagogiques-fle/mexique-unchewing-gum-biodegradable

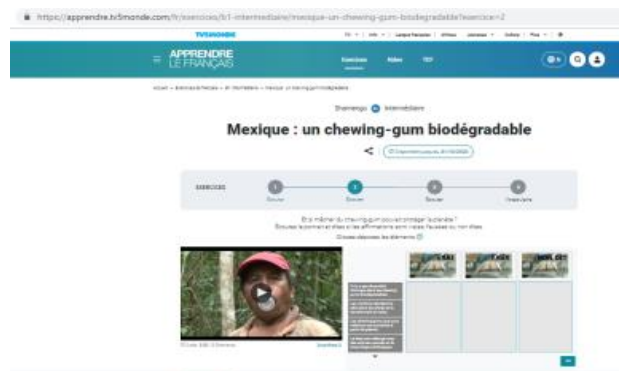

Image 2. Mexique: un chewing-gum biodegradable. Exercices de français: B1 intermédiaire.

Url: https://apprendre.tv5monde.com/fr/exercices/b1-intermediaire/mexique-unchewing-gum-biodegradable? exercice $=2$ 


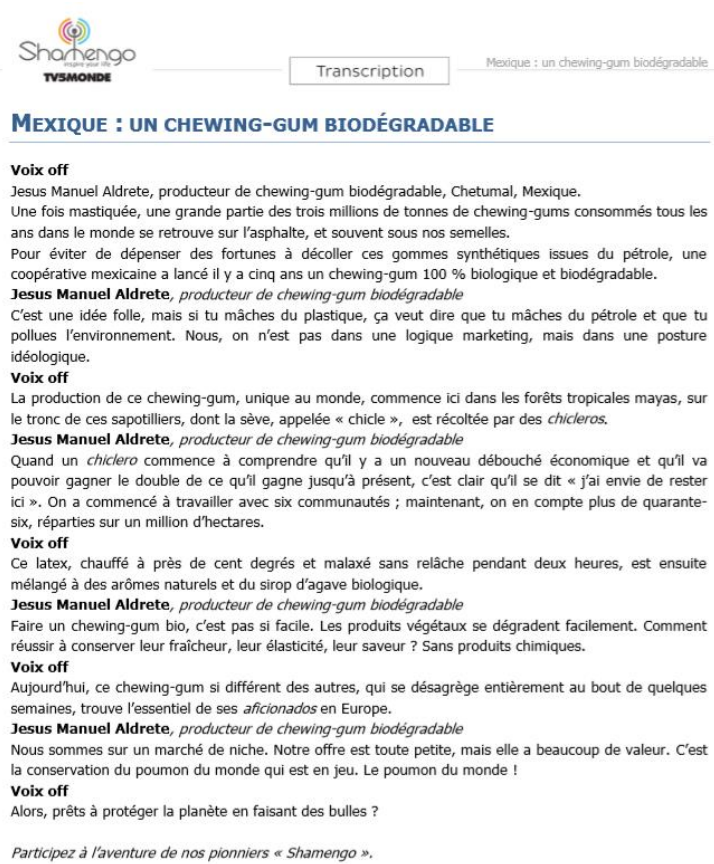

Image 3. Texte:Mexique: un chewing-gum biodegradable.

\section{Étape 4}

Les étudiants sont encouragés à préparer leur propre texte et à réaliser une vidéo basée sur ce texte. Pour un travail plus productif, on fait attention à ce plan : 1. Problème environnemental dans le monde ; 2 . Problème environnemental en Russie ; 3 . Déchets ; 4. Pollution de l'air ; 5. Pollution de l'eau ; 6. Espèces menaces ; 7. Destruction des ressources naturelles.

\section{Étape 5}

Montage et téléchargement de video faite sur Youtube. Présentation du projet.

Ainsi, on voit que la création de vidéos éducative contribue non seulement à la capacité de naviguer dans une grande quantité d'informations, mais éduque également l'étudiant, lui apprend à penser de manière créative, favorise la maîtrise de l'information.

Ainsi, la compétence médiatique, formée dans le processus de création et d'application d'informations vidéo, donne aux enseignants une idée dont les textes médiatiques, faisant partie de la vie quotidienne, aident à comprendre le monde qui nous entoure, l'environnement riche en informations, dans les diverses variations sociales, les positions économiques et politiques, affecter le niveau de compétence médiatique des étudiants.

\section{Conclusion}

L'une des tâches principales de l'éducation moderne est d'enseigner aux étudiants à naviguer dans un large flux d'informations et à leur enseigner l'éducation aux médias.

L'un des moyens les plus productifs de former la compétence médiatique des étudiants consiste à utiliser et à créer leurs propres vidéos éducatives. 
La compétence médiatique des étudiants s'exprime sous forme d'indicateurs de contact, de motivation, de conception, d'évaluation et de création. Le problème de la formation de compétence médiatique des étudiants est particulièrement pertinente dans nos jours, dès lors que des TICs développent plus rapide que possible. Elle permet aux étudiants de naviguer dans le monde des médias, de percevoir, d'analyser, d'évaluer et de mettre en pratique de manière adéquate les données obtenues des médias, d'Internet, de films vidéo, etc.

L'utilisation de matériel vidéo en classe du FLE est de plus en plus populaire car elle contribue à l'actualisation des connaissances et au maintien d'une motivation interne à apprendre le FLE. Le matériel médiatique peut être utilisé en classes du FLE ainsi qu'en classes complémentaires.

Les supports médiatiques créés par des étudiants, utilisés en classes du FLE doivent quand meme être authentiques, alors que de nature pédagogique et liés au processus d'apprentissage.

La formation de compétence médiatique des adolescents en tant que guide de l'espace informationnel est le processus d'interaction pédagogique le plus important et le résultat de l'éducation aux medias. Donc, c'est une priorité dans le domaine du développement culturel et pédagogique de la génération de la jeunesse moderne. La formation de la compétence médiatique de l'individu est pertinente et demandée dans une société riche en informations avec un accès ouvert à toutes les informations des médias dans l'espace mondial de l'information ou de la société avec de futurs changements radicaux dans la sphère éducative, avec des approches convergentes et interculturelles des nouveaux médias pour l'organisation créative du processus éducatif et cognitif.

L'importance théorique de la recherche réside dans le fait que le contenu de la notion de compétence médiatique est révélé, ainsi que la possibilité d'utiliser des supports médiatiques pour former la compétence médiatique des étudiants en classes du FLE dans la pratique de l'enseignement supérieur.

L'importance pratique en est qu'on propose un algorithme pour l'organisation du travail indépendant des étudiants dans le processus de création de supports pédagogiques médiatiques lorsqu'ils étudient le FLE dans des établissements d'enseignement supérieur.

\section{Bibliographic references}

ARUTYUNOVA, ZH.M. 2019. Voyage au pays de la lecture. Récits d'écrivains contemporains de langue française (niveau avancé).Traveling to the reading country. Stories of modern francing language writers (advanced stage). In: A study guide on the French language using original texts to develop reading and speaking skills. Moscow. Languages of the Peoples of the World. 124 p. ISBN 978-5-6042214-1-9. Available online: https://elibrary.ru/item.asp?id=37155602

BABUSHKINA, L.E. - BALAKISHIYEVA, V. 2017. K voprosu o formirovanii mediakompetentnosti studentov pedagogicheskogo vuza [On the question of the media competence formation of pedagogical institution students] Evsevievskiye chteniya. Seriya: Lingvistika i metodika: mezdistsiplinarniy podhod: sbornik nauchnih trudov, Saransk. pp. 58-63. Available online: https://elibrary.ru/item.asp?id=32566967

BABUSHKINA, L.E. - KADAKIN, V.V. - SHUKSHINA, T.I. - FALILEEV, A.E. PISKUNOVA, S.I. 2017.Technology of linguistic competence formation of students in pedagogical institution. In: Ponte, vol. 73, n. 2, pp. 109-118. Available online: http://www.pontejournal.net/mainpanel/abstract.php?TOKEN=gRkgF5411G\&PID=P J-D56W9

BARANOV, O.A. 2002. Mediaobrazovanie v shkole i vuze [Media education at school and university]. Tver': Izdatel'stvo Tverskogo gosudarstvennogo pedagogicheskogo universiteta. $87 \mathrm{p}$. 
BARIEV, A K. - KIREEVA, Z.R. - ZHOU, N. - KADI, S. 2018. The overcoming the communication barriers of students as means of a personalization of education. In: Journal of Social Studies Education Research, vol. 9, n. 3, pp. 398-409. Available online: https://elibrary.ru/item.asp?id=35720853

BIM, I.L. 1988. Teoriya i praktika obucheniya nemeckomu yazyku v srednej shkole: problemy i perspektivy: uchebnoe posobie [Theory and practice of German teaching in secondary school: problems and prospects: a manual]. Moscow: Prosveshchenie, $256 \mathrm{p}$.

BIROVA, J. - OCOVAY, J. - VASBIEVA, D.G. 2016.Implementation of New Teaching Technologies during the Action Research by Experienced Language Teachers. In: IEJME: Mathematics Education. vol. 11, n. 8, pp. 3089-3103. ISSN: 2468-4945.

BIROVA, J. - VASBIEVA, D.G. - MASALIMOVA, A.R.2017. Communication in French Foreign Language Learning by Implementing the Aspects of Interculturality. In: Komunikacie. vol. 19, n.4, pp. 95-104. ISSN: 1335-4205.

BORISOVA, O.V. - VASBIEVA, D.G. - MALYKH, N.I. - VASNEV, S.A. 2016. Problem of Using Innovative Teaching Methods for Distance Learning Students.In: IEJME: Mathematics Education. vol. 11, n. 5, pp. 1175-1184. ISSN: 2468-4945.

CHEVALLIER, J. 1980. Cine-club et action educative. Paris: CNDP. 64 p.

FEDERMAN, J. 1997. National Television Violence Study. Santa Barbara: Center for Communication and social Policy University of California. $54 \mathrm{p}$.

FEDOROV,A. V. 2005. Mediaobrazovanie, mediagramotnost', mediakritika i mediakul'tura. [Media education, media literacy, media criticism and media culture] In: Vysshee Obrazovanie v Rossii [Higher Education in Russia]. n 6. pp.134-138. Available online: http://vovr.ru/upload/6-05.pdf

FREINET, C. 1994. Oeuvres pedagogiques.Paris : Seuil. T. 1. 591 p.

GNEZDILOVA, E.V. - ZAITSEV, А.А. 2018. Рольмедиатекставформиро

ваниикартинымирасовременногочеловека / The role of mediatext in the formation of the picture of the modern world. In: Gumanitarnye Nauki I Obrazovanie [The Humanities and Education], vol. 9, n. 2, pp. 146-151. Available online: https://elibrary.ru/item.asp?id=35451673

GUREVICH, P.S. 2011.Culturology. Moscow: KNORUS. 448 p.

HUTORSKOJ,A.V. 2002. Klyuchevye kompetencii kak komponent lichnostnoorientirovannoj paradigmy obrazovaniya [Key competencies as a component of the student-centered education paradigm].Narodnoe obrazovanie [National Education]. n. 2. pp. 54-68. ISSN: 0130-6928.

KADAKIN, V.V. - SHUKSHINA, T.I. - PISKUNOVA, S.I. - BABUSHKINA, L.E. - FALILEEV, A.E. 2016. Pedagogical Conditions of Multilevel Foreign Languages Teaching in Pedagogical Higher Education. In: International Journal of Environmental and Science Education, vol. 12, n. 14, pp. 6603-6615. Available online: http://www.ijese.net/makale/945

KASHCHUK, S. 2019. Competences representing the goal of foreign language learning: is the approach real? [Les competences en objectif d'apprentissage des langues: mythe ou realite?]. In: XLinguae, vol. 12, n. 1XL, pp. 40-49. ISSN 13378384, eISSN 2453-711X, DOI: 10.18355/XL.2019.12.01XL.04

KOSTINA, E.V. - PRONKINA, V.M. 2015. Tests as the modern means of assessment of the students' progress in learning English. In: Gumanitarnye Nauki I Obrazovanie [The Humanities and Education], vol. 22, n. 2, pp. 91-93. Available online: http://elibrary.ru/download/94457070.pdf

KOZARENKO, O.M. 2019. Evaluation criteria for research work of students in a foreign language and the principles of their development [Критерииоценкиисследовательскойработыстудентовнаиностранномязыкеиприн

XLinguae, Volume 13 Issue 1, January 2020, ISSN 1337-8384, eISSN 2453-711X 
ципыихразработки]. In: XLinguae, vol. 12, п. 2, pp. 195-209. ISSN 1337-8384, eISSN 2453-711X, DOI: 10.18355/XL.2019.12.02.16

LAZUTOVA, L.A. - FALILEEV, A.E. 2016. Using of Google resources for formation students' lexical skills teaching foreign languages at language faculties. In: Gumanitarnye Nauki I Obrazovanie [The Humanities and Education], vol. 26, n. 2, pp. 96-99. Available online: http://elibrary.ru/download/39734170.pdf

LEVINA, E.A. - KOSTINA, E.V. 2016. Using educational podcasts to teach the bachelors speaking in foreign language. In: Gumanitarnye Nauki I Obrazovanie [The Humanities and Education], vol. 26, n. 2, pp. 99-102. Available online: http://elibrary.ru/download/21198861.pdf

MASALIMOVA, A.R. - KORZHUEV, A.V. - VASBIEVA, D.G. - GALUSHKIN, A.A. - MAIDANGALIEVA, ZH.A. - ZAITSEVA, N.A. 2019. Preservation of National Languages as a Factor of National Security in the Contemporary WorldIn: European Journal of Science and Theology. vol. 15, n. 2, pp. 113-121. ISSN: 18410464.

MUZYKANT, V.L. - PONOMARENKO, E.B. - BARABASH, V.V. DENISENKO, V.N. - SHLYKOVA, O.V. 2019. New media: invective language transformation of global communication. In: XLinguae, vol. 2, n.1, ISSN 1337-8384, eISSN 2453-711X, pp. 80-90. DOI: 10.18355/XL.2019.12.01.06

SAIENKO, N. - KALUGINA, O.A. - BAKLASHOVA, T.A. - RODRIGUEZ, R.G. 2019. A stage-by-stage approach to utilizing news media in foreign language classes at higher educational institutions. In: XLinguae, vol. 12, n.1, pp. 91-102. DOI: 10.18355/XL.2019.12.01.07.

http://xlinguae.eu/2019_12_01_07.html

Available

online:

SHUKSHINA, T.I. - BABUSHKINA, L.E. 2014. Formation of sociocultural competence among students of a pedagogical higher education institution using ICT when learning foreign languages. In: Life Science Journal, vol. 11, n.6, pp. 565-568. ISSN: 2372-613X.

SHUKSHINA, T.I. - BUYANOVA, I.B. - GORSHENINA, S.N. - NEYASOVA, I.A. 2016. Experience of Testing Practice-Oriented Educational Model of Pedagogical Master's Program. In: International Journal of Environmental and Science Education, vol. 11, n. 14, pp. 6482-6492. Available online: http://www.ijese.net/makale/934

SMIRNOVA, N.V. - BAGRAMOVA, N.V. 2018. An empirical investigation on undergraduate students' writing skills in a foreign language as a social practice within a non-linguistic university. In: Novosibirsk state pedagogical university bulletin, vol. 8, n. 6., pp. 201-215. ISSN: 2226-3365. Available online: https://elibrary.ru/item.asp?id=36655301

SOLNTSEVA, A.V. 2019. French as foreign language: how to increase students' motivation? In: The stages of romanian languages development: from the language of live communication to the national language. In: Interuniversity collection of scientific papers. Executive editor Skuratov I.V. Moscow. pp. 176-179. Available online: https://elibrary.ru/item.asp?id=38528194

VETOSHKIN,A.A. 2015. English as a means of integration into the international education space. In: Gumanitarnye Nauki I Obrazovanie [The Humanities and Education], vol. 24, n. 4, pp. 100-102. Available online: http://elibrary.ru/download/55244119.pdf

YAKUNCHEV, M. - GORSHENINA, S. 2013. Diagnostics of Ethno-Cultural Competence of the Future Teachers. In: Middle-East Journal of Scientific Research, vol. 16, n. 12, pp. 1709-1713. Available online:https://www.idosi.org/mejsr/mejsr16(12)13/17.pdf

ZIMNYAYA, I.A. 2003.Klyuchevye kompetencii - novaya paradigma rezul'tata obrazovaniya [Key competencies - a new paradigm of the education result]. In: Higher Education Today. n 5. pp. 20-26. ISSN 1726-667X. 
ULIL, A.N. - SAHRIL, M.H. 2018. Strategies to support foreign language learning in large classes: a systematic literature review. In: XLinguae, vol. 11, n. 1, pp. 3-15. ISSN 1337-8384.

Words: 9449

Characters: 67580 (21 standard pages)

Assoc. Prof. Larisa E. Babushkina, PhD of Pedagogy

Department of Foreign Languages and Teaching Methods

Mordovian State Pedagogical Institute

Studencheskaya Str., 11A

470007 Saransk

Russia

lb_77@list.ru

Assoc. Prof. Olga A. Kalugina

Department of Foreign Languages

Financial University under the Government of the Russian Federation

Leningradsky prospect 49

125993 Moscow

Russia

kaluginaruc@mail.ru 